\title{
68Ga-PSMA-11 PET/MR Can Be False Positive in Normal Prostatic Tissue
}

Ferraro, Daniela A ; Rupp, Niels J ; Donati, Olivio F ; Messerli, Michael ; Eberli, Daniel ; Burger, Irene A

\begin{abstract}
Prostate-specific membrane antigen (PSMA) is a transmembrane glycoprotein expressed in the cytosol of normal prostate tissue and highly overexpressed on the membrane of prostate cancer, therefore increasingly used to image prostate cancer. We report a case of a 65-year-old man with two focal PSMApositive areas on a Ga-PSMA-11 PET/MR, one corresponding to a prostate carcinoma (Gleason score $4+$ 3 ) and another region without any evidence of malignancy, but with corresponding high PSMA-expression on immunohistochemistry.
\end{abstract}

DOI: https://doi.org/10.1097/RLU.0000000000002473

Posted at the Zurich Open Repository and Archive, University of Zurich ZORA URL: https://doi.org/10.5167/uzh-165994

Journal Article

Published Version

Originally published at:

Ferraro, Daniela A; Rupp, Niels J; Donati, Olivio F; Messerli, Michael; Eberli, Daniel; Burger, Irene A (2019). 68Ga-PSMA-11 PET/MR Can Be False Positive in Normal Prostatic Tissue. Clinical Nuclear Medicine, 44(4):e291-e293.

DOI: https://doi.org/10.1097/RLU.0000000000002473 


\title{
${ }^{68}$ Ga-PSMA-11 PET/MR Can Be False Positive in Normal Prostatic Tissue
}

\author{
Daniela A. Ferraro, MD, * Niels J. Rupp, MD, † Olivio F. Donati, MD, $\neq$ Michael Messerli, MD, * \\ Daniel Eberli, MD, PhD, $\S$ and Irene A. Burger, MD*
}

\begin{abstract}
Prostate-specific membrane antigen (PSMA) is a transmembrane glycoprotein expressed in the cytosol of normal prostate tissue and highly overexpressed on the membrane of prostate cancer, therefore increasingly used to image prostate cancer. We report a case of a 65 -year-old man with two focal PSMA-positive areas on a ${ }^{68}$ Ga-PSMA-11 PET/MR, one corresponding to a prostate carcinoma (Gleason score $4+3$ ) and another region without any evidence of malignancy, but with corresponding high PSMAexpression on immunohistochemistry.
\end{abstract}

Key Words: ${ }^{68} \mathrm{Ga}-\mathrm{PSMA}$, PET/MR, false positive, prostate cancer

(Clin Nucl Med 2019;44: e291-e293)

Received for publication August 14, 2018; revision accepted December 16, 2018. From the *Department of Nuclear Medicine, University Hospital Zürich, University of Zürich; †Department of Pathology and Molecular Pathology, University Hospital and University of Zürich; Institute of Diagnostic and Interventional Radiology, University Hospital Zürich; and §Department of Urology, University Hospital Zürich, University of Zürich, Zürich, Switzerland. Conflicts of interest and sources of funding: none declared.

Correspondence to: Daniela A. Ferraro, MD, Department of Nuclear Medicine, University Hospital Zürich, Rämistrasse 100, 8091 Zürich, Switzerland. E-mail: Daniela.ferraro@usz.ch.

Copyright (C) 2019 Wolters Kluwer Health, Inc. All rights reserved.

ISSN: 0363-9762/19/4404-e291

DOI: $10.1097 /$ RLU.0000000000002473

\section{REFERENCES}

1. Luque RJ, Lopez-Beltran A, Perez-Seoane C, et al. Sclerosing adenosis of the prostate. Histologic features in needle biopsy specimens. Arch Pathol Lab Med. 2003;127:e14-e16.

2. Chang A, Amin A, Gabrielson E, et al. Utility of GATA3 immunohistochemistry in differentiating urothelial carcinoma from prostate adenocarcinoma and squamous cell carcinomas of the uterine cervix, anus, and lung. Am J Surg Pathol. 2012;36:1472-1476.

3. Haffner MC, Kronberger IE, Ross JS, et al. Prostate-specific membrane antigen expression in the neovasculature of gastric and colorectal cancers. Hum Pathol. 2009;40:1754-1761.

4. Chang SS, O'Keefe DS, Bacich DJ, et al. Prostate-specific membrane antigen is produced in tumor-associated neovasculature. Clin Cancer Res. 1999;5: 2674-2681.

5. Siva S, Callahan J, Pryor D, et al. Utility of ${ }^{68}$ Ga prostate specific membrane antigen-positron emission tomography in diagnosis and response assessment of recurrent renal cell carcinoma. J Med Imaging Radiat Oncol. 2017;61: 372-378.

6. Sasikumar A, Joy A, Nanabala R, et al. (68)Ga-PSMA PET/CT imaging in primary hepatocellular carcinoma. Eur J Nucl Med Mol Imaging. 2016;43: 795-796.

7. Sasikumar A, Joy A, Pillai MR, et al. Diagnostic value of 68Ga PSMA-11 PET/CT imaging of brain tumors - preliminary analysis. Clin Nucl Med. 2017;42:e41-e48.

8. Lütje S, Gomez B, Cohnen J, et al. Imaging of prostate-specific membrane antigen expression in metastatic differentiated thyroid cancer using 68Ga-HBED-CC-PSMA PET/CT. Clin Nucl Med. 2017;42:20-25.

9. Sathekge M, Lengana T, Modiselle M, et al. 68Ga-PSMA-HBED-CC PET imaging in breast carcinoma patients. Eur J Nucl Med Mol Imaging. 2017; 44:689-694.

10. Hofman MS, Hicks RJ, Maurer T, et al. Prostate-specific membrane antigen PET: clinical utility in prostate cancer, normal patterns, pearls, and pitfalls. Radiographics. 2018;38:200-217.

11. Kobe C, Maintz D, Fischer T, et al. Prostate-specific membrane antigen PET/CT in splenic sarcoidosis. Clin Nucl Med. 2015;40:897-898.

12. Pizzuto DA, Muller J, Muhlematter U, et al. The central zone has increased ${ }^{68}$ Ga-PSMA-11 uptake: "Mickey Mouse ears" can be hot on ${ }^{68}$ Ga-PSMA11 PET. Eur J Nucl Med Mol Imaging. 2018;45:1335-1343. 

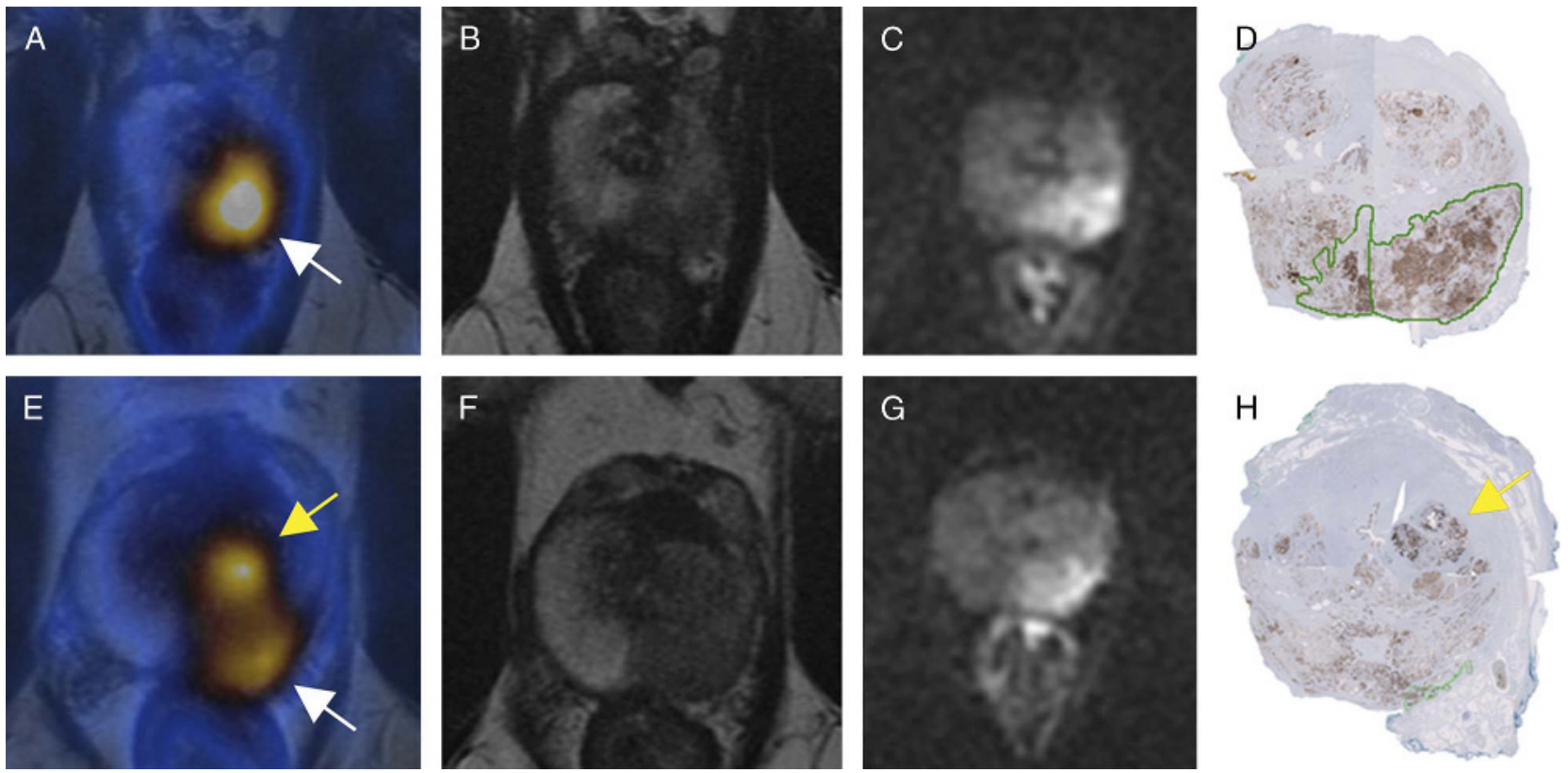

FIGURE 1. A 65 -year-old man with prostate adenocarcinoma (PSA: $7.8 \mathrm{ng} / \mathrm{ml}$ and Gleason Score: $4+3=7$ on biopsy), was referred for a ${ }^{68} \mathrm{Ga}$-PSMA-11 positron emission tomography/magnetic resonance imaging $\left({ }^{68} \mathrm{Ga}-\mathrm{PSMA}-11 \mathrm{PET} / \mathrm{MR}\right)$ for staging before radical prostatectomy. On PET/MR two focal areas in the prostate gland had intense ${ }^{68} \mathrm{Ga}-\mathrm{PSMA}-11$ accumulation. PET/MR fusion image (A) shows intense PSMA-activity in the lesion located in the left paramedian peripheral zone, from base to apex (white arrow), with T2-weighted hypointense signal on the axial T2 weighted MR sequence (B) and diffusion restriction on the axial diffusion weighted images (DWI, $b=1000)(C)$. On histopathology (D) the prostate adenocarcinoma had a high PSMA-expression on immunohistochemistry (green margin). A second focal area with intense PSMA-activity on fused axial PET/MR image (E), was localized in the left midglandular transition zone (yellow arrow) anteriorly to the tumor in the peripheral zone (white arrow), without corresponding abnormality seen on axial T2 weighted MR sequence $(\mathbf{F})$, or DWI (G). On histopathology $(\mathbf{H})$ no malignancy was detected, despite a prominent overexpression of PSMA on immunohistochemistry (yellow arrow). 

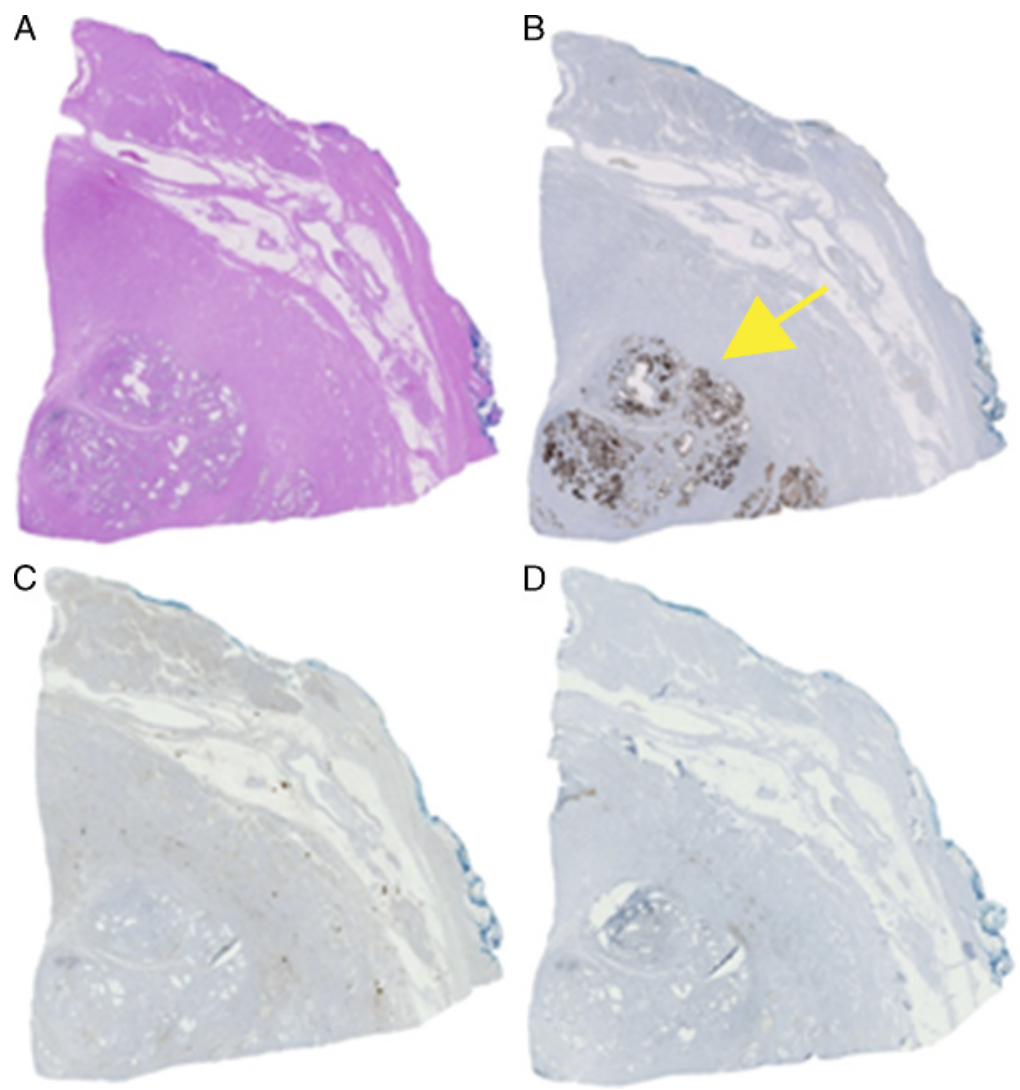

FIGURE 2. To exclude other specific histological abnormalities such as urothelial metaplasia or sclerosing adenosis in the PSMA-overexpressing area in the transition zone, further immunohistochemistry was performed. On histopathology, the transition zone tissue on hematoxylin and eosin staining (HE) (A) did not show any sign of malignancy. On PSMA immunohistochemistry, however, a high PSMA-expression was seen (yellow arrow), with cytoplasmic and membranous expression (B). S-100 did not show an atypical expression pattern (C), excluding a sclerosing adenosis. ${ }^{1}$ (D) GATA-3 only showed minimal focal expression, corroborating that there is no significant urothelial metaplasia. ${ }^{2}{ }^{68} \mathrm{Ga}$-PSMA is known to be overexpressed in nonprostatic tumor neovasculature, ${ }^{3,4}$ in several malignancies, ${ }^{5-9}$ as well as in benign conditions like inflammation, osteoblastic processes and benign tumors. ${ }^{10,11}$ These potential pitfalls should be carefully taken into consideration to avoid misinterpretation of ${ }^{68} \mathrm{Ga}$-PSMA-11 PET whole body scans. In the prostate gland, however, ${ }^{68}$ Ga-PSMA-11 PET is still considered very specific for primary prostatic malignancy, without any false positive reports. A potential pitfall of mild, physiological increased ${ }^{68} \mathrm{Ga}$-PSMA-11 accumulation on PET imaging in the central zone was recently described. ${ }^{12}$ However, with the presented case, we show that also normal prostatic tissue in the transition zone can present with focal PSMA overexpression on cell membrane, without any signs of malignant transformation, benign tumors, other malignancy (urothelial cancer) or inflammatory changes. This unusual presentation of normal glandular prostatic tissue has the potential to lead to false positive findings on ${ }^{68} \mathrm{Ga}$-PSMA-11 PET in patients referred for prostate cancer staging. Therefore, in the setting of primary evaluation of prostate cancer not only careful investigation regarding the sensitivity and detection rate is mandatory, but also the cautious evaluation of the specificity to improve the understanding and accurate interpretation of physiological ${ }^{68}$ Ga-PSMA-11 accumulation in the prostate. 